\title{
Estimation of Intermodulation Rejection Value as a Function of Frequency in Power Amplifier Using AM-AM and AM-PM Diagrams Based on Power Series Analysis
}

\author{
Aazar Saadaat Kashi*, Mahmoud Kamarei, Mohsen Javadi \\ Department of Electrical and Computer Engineering, University of Tehran, Tehran, Iran \\ Email: ${ }^{*}$ a.kashi@ut.ac.ir
}

Received May 4, 2012; revised June 4, 2012; accepted June 11, 2012

\begin{abstract}
A method to predict intermodulation (IM) products of two tone test based on Amplitude to amplitude (AM-AM) and amplitude to phase (AM-PM) diagrams of power amplifier is proposed in this paper. An RF power amplifier is mathematically modeled by a power series in order of 13 . Coefficients of the transfer function are obtained by odd-order polynomial fitting of the transfer function of the power amplifier that is modeled by power series, with AM-AM and AM-PM diagrams. Because of considering AM-PM distortion, coefficients have become complex. By using this transfer function, analytical expressions of IM products are derived. Frequency effect of IM products are modeled in suggested method to estimate the effects of changing in input frequency on output. With the mean of this factor the model is able to predict IM products of wideband frequency input. Simulated results agree well with the predicted method in comparisons.
\end{abstract}

Keywords: Intermodulation Product; Two Tone Test; RF Power Amplifier; Power Series; AM-AM and AM-PM Diagrams

\section{Introduction}

In modern communication systems, digital communication schemes, such as code division multiple access, where the information is carried are admitted by various systems. The purpose that makes CDMA spectrally efficient and popular in recent digital mobile communication systems lies in allocation a unique code for each user, so a certain number of users can communicate at the same time and frequency.

Although, the high spectral efficiency gained by using a CDMA scheme, it degrade at the cause of spectral regrowth that is inevitably generated when signal is passed through nonlinear devices of a RF transmitter.

As in other communication systems, one of the critical and costly components in digital cellular communication systems is RF power amplifier. The power amplifier is the major source of nonlinearity in a communication system. To increase their efficiency, power amplifiers are sometimes driven into their nonlinear region [1], thus causing spectral regrowth, increasing bit error rate, interference to adjacent channel, and intermodulation products to be generated. In extreme these IM products can interfere with signals that are being amplifies [2].

${ }^{*}$ Corresponding author.
Since the spectral regrowth is stringently regulated and is mostly generated by a nonlinear RF power amplifier [3], it is very important for RF system designers to predict the distortion effects of power amplifiers and analyze its effect on the output.

Given certain power amplifier characteristics, it is desirable to be able to predict, without running time-domain simulations, whether the power amplifier can be used to amplify certain type of signals, i.e. the amount of spectral regrowth is within limits [4]. This is due to the fact that channel impairments like the intermodulation distortion can be readily estimated with the knowledge of the nonlinear characteristics of the power amplifier, and properly modeled it [5].

Since, third-order polynomials for modeling the nonlinearities are suitable for weakly nonlinear systems [6], this paper presents an analytical expression that predicts IM products of the output of power amplifier. With the mean of power series transfer function (in order of 13) and AM-AM and AM-PM distortions of power amplifier, it modeled and analyzed to predict first, third, and fifth order IM products of the output.

One of the advantages of the presented model is that it uses both AM-AM and AM-PM diagram to characterize power amplifier transfer function. The phase variations 
have an important effect on the spectral regrowth [7]. However, similar previous models only used AM-AM diagram to characterize power amplifier's transfer functions [3,8-11] while such an effect is considered in [7,1215]. Although, in this case, transfer function's coefficients have become complex and this increases the difficulty of the calculations, but neglecting the AM-PM distortion causes many problems in predicting output spectrum [16-18]. Considering variation in output response of power amplifier with disparity on input frequency is the major factor that is deliberated in our model. It is found that adjacent channel power ratio performances is not only dependent on the input signal magnitude (or power) but also dependent on the bandwidth of the signal that is used [19]. Main advantage of this model is that we don't neglect the frequency dependence of power amplifier's transfer function coefficient that makes asymmetric spectrum in the upper and lower band which is not taking into consideration in $[3,7,14,15]$. The factor is added in our model with considering our transfer function's constants as a function of input frequency besides they are complex. With considering frequency effect, this model is able to be used for wide band frequency range inputs such as UWB systems, WCDMA input, and input signals with complexity in their phase or frequency. With the mean of this method we also can analyze power amplifier systems in the case that their circuit is not reachable.

\section{Power Series Model of Power Amplifier}

Estimating a perfect nonlinear model of power amplifier that can describe output characteristics is of the great importance. In this part power series model of power amplifier is described, and with using this model and AM-AM and AM-PM diagrams, output IM products will be predicted.

Generally speaking, a practical amplifier is only a linear device in its linear region, meaning that the amplifier output will not exactly a scaled copy of the input signal when the amplifier works beyond linear region [9].

Considering an amplifier as a function box it can be modeled by a power series $[20,21]$. The input signal is of the form:

$$
x(t)=A(t) \cdot \cos \left(w_{c} t+\theta(t)\right)=\operatorname{Re}\left[\tilde{x}(t) \cdot e^{j \theta(t)} \cdot e^{j \omega_{c} t}\right]
$$

where $A(t)$ is an amplitude and $\theta(t)$ is a phase of carrier, $\omega_{c}$ is the carrier angular frequency, and $\tilde{x}(t)$ denotes the base band equivalent input signal.

Because of nonlinearities of the power amplifier that can represent by AM-AM and AM-PM distortion the amplitude and phase of the output are affected, respectively.

The output can represent as follow [7]:

$$
\begin{aligned}
y(t) & =\operatorname{Re}\left[\tilde{y}(t) \cdot e^{j \theta(t)} \cdot e^{j \omega_{c} t}\right] \\
& =\operatorname{Re}\left[F(A(t)) \cdot e^{j \theta(t)} \cdot e^{j \omega_{c} t}\right] \\
& =|F(A(t))| \cdot \cos \left(\omega_{c} t+\theta(t)+\angle F(A(t))\right)
\end{aligned}
$$

where $y(t)$ represents the equivalent output signal and $F(A(t))$ denotes the complex envelope transfer function of the power amplifier. Function $F$ can be represented by a power series or by an orthogonal function expansion or perhaps reasonably well approximated over the range of $x(t)$ by a polynomial in $x$ [22]. With considering power series model and using baseband equivalent model for power amplifier, we can represent $F$ as:

$$
\begin{aligned}
y(t) & =F(x(t))=\sum_{n}^{N} a_{n} x^{n}(t) \\
x(t) & =\operatorname{Re}\left[\tilde{x}(t) \cdot e^{j \theta(t)} \cdot e^{j \omega_{c} t}\right] \\
& =\frac{1}{2}\left[\tilde{x}(t) \cdot e^{j \theta(t)} \cdot e^{j \omega_{c} t}+\tilde{x}^{*}(t) \cdot e^{-j \theta(t)} \cdot e^{-j \omega_{c} t}\right]
\end{aligned}
$$

By using the binomial expansion for $x^{n}(t)$ and considering only the first zone components we obtain [22]:

$$
\begin{aligned}
& x^{n}(t)=\frac{1}{2^{n}} \sum_{k=0}^{n} C_{n}^{k}[\tilde{x}(t)]^{k}\left[\tilde{x}^{*}(t)\right]^{n-k} e^{\left(j \omega_{c} t+j \theta(t)\right)(2 k-n)} \\
& =\frac{1}{2^{n-1}} C_{n}^{\frac{n+1}{2}}\left|\tilde{x}^{*}(t)\right|^{n-1} \tilde{x}(t) \text { for } n \text { : odd }
\end{aligned}
$$

The complex envelope of the first-zone component of $y(t)$ is:

$$
\tilde{y}(t)=\tilde{x}(t) \sum_{m=0}^{\frac{N-1}{2}} \frac{a_{2 m+1}}{2^{2 m}} C_{2 m+1}^{m+1}\left|\tilde{x}^{*}(t)\right|^{2 m}
$$

With simplify Equation (6), we can write the base band output signal as follow if we assume that the $\theta(t)$ (phase of carrier) is zero:

$$
\tilde{y}(t)=\sum_{n=1}^{N} a_{n} \tilde{x}^{n}(t) \quad n \text { : odd }
$$

In power amplifier transfer function's coefficients characterization, if only AM-AM distortion is considered the coefficients that are obtained from the fitting of AM-AM diagram and proposed transfer function, have become real. Also neglecting the AM-PM distortion causes many problems in predicting IM products and then output spectrum.

In this model, the coefficients $a_{n}$ would be obtained by fitting a polynomial of degree $N$ to AM-AM and AM-PM diagrams of power amplifier that obtained by simulation of power amplifier in ADS. The coefficient $a_{n}$ considered to be complex, because of using AM-PM distortion besides AM-AM. To obtain a good fit we would require a large value of $N$, which would reduce the efficiency of 
such model [22]. In this work we only consider first $13^{\text {th }}$ terms of power series.

\section{Predicting IM Products with the Mean of Power Amplifier Model}

When multiple signals are passed through a common amplifier, the nonlinearity of amplifier cause intermodulation (IM) products to be generated [2]. One of the major areas in analyzing nonlinear effect of power amplifier is considering variation in output response of power amplifier with disparity on input frequency. Variation in IM product with frequency is deliberate in our model.

With considering frequency effect, the method is able to predict IM products of every type of input such as WCDMA or input signals with complexity in their phase or frequency. Also, it can use to analyze power amplifiers system when their circuit details are not reachable.

Generally, IM3 is used as a linearity parameter, but when an input signal becomes large, higher order IM products are also generated [3]. So, by using our model, an analytical expression that relates IM product in general order to the amplitude of the input of two tone test is derived.

Input signal of the two tone test can be considered as follow:

$$
\begin{aligned}
x(t) & =\frac{s}{2} \cos \left[\left(\omega_{c}+\Delta \omega\right) t\right]+\frac{s}{2} \cos \left[\left(\omega_{c}-\Delta \omega\right) t\right] \\
& =s \cos (\Delta \omega t) \cos \left(\omega_{c} t\right)=\operatorname{Re}\left[s \cos (\Delta \omega t) \cdot e^{j \omega_{c} t}\right]
\end{aligned}
$$

where $s / 2$ is an amplitude of each tone. By comparing this equation with Equation (1), we can represent the baseband input signal as:

$$
\tilde{x}(t)=s \cos (\Delta \omega t)
$$

The baseband output signal with respect to Equation (8) can be represented as follow [7]:

$$
\begin{aligned}
\tilde{y} & (t)=\sum_{n=1}^{N} a_{2 n-1} \tilde{x}^{2 n-1}(t)=\sum_{n=1}^{N} a_{2 n-1} s^{2 n-1} \cos ^{2 n-1}(\Delta \omega t) \\
= & {\left[a_{1} s+\frac{3}{4} f_{3} s^{3}+\cdots+\frac{C_{N-1}^{2 N-1}}{4^{N-1}} a_{2 N-1} s^{2 N-1}\right] \cos (\Delta \omega t) } \\
& +\left[\frac{1}{4} f_{3} s^{3}+\cdots+\frac{C_{N-2}^{2 N-1}}{4^{N-1}} a_{2 N-1} s^{2 N-1}\right] \cos (3 \Delta \omega t)+\cdots \\
& +\left[\frac{1}{4^{N-1}} a_{2 N-1} s^{2 N-1}\right] \cos ((2 N-1) \Delta \omega t) \\
= & \tilde{y}_{1} \cos (\Delta \omega t)+\tilde{y}_{3} \cos (3 \Delta \omega t)+\tilde{y}_{5} \cos (5 \Delta \omega t)+\cdots \\
& +\tilde{y}_{2 N-1} \cos ((2 N-1) \Delta \omega t) \\
= & \sum_{n=1}^{N} \tilde{y}_{2 n-1} \cos ((2 n-1) \Delta \omega t)
\end{aligned}
$$

where $\tilde{y}_{2 n-1}$ is an output complex envelope of $\mathrm{IM}_{2 n-1}$.
So we can write an analytical expression for $\mathrm{IM}_{2 n-1}$ as a function of amplifier characteristics and input amplitude "s", as follow:

$$
\tilde{y}_{2 n-1}=\sum_{j=n}^{N} \frac{C_{j-n}^{2 j-1}}{4^{j-1}} a_{2 j-1} s^{2 j-1}
$$

\section{Simulation Results}

Verify our derivation, a simulation of an RF power amplifier with ADS simulator is performed. The carrier frequency is $850 \mathrm{MHz}$. To predict the IM products, power amplifier's transfer function coefficients that are the function of input frequency must be obtained first. To evaluate these coefficients $\left(a_{2 n+1}\right)$ the amplifier is simulated with one-tone input in ADS and the input voltage is swept in its range to AM-AM and AM-PM diagrams are obtained.

Amplitude to amplitude and amplitude to phase distortions are two distortion effects in power amplifiers at high output power levels, causing out of band interference in the transmitted signal and a bit errors in the received signal [23]. Using diagrams and our analytical equation for the output, complex coefficients $a_{2 n+1}$ will be found by odd order polynomial fitting that perform in MATLAB. Power amplifier's coefficients must show its nonlinearity, so, we should simulate our circuit both in linear and nonlinear region. On the other hand, input voltage must sweep to voltages that make the circuit being in its nonlinear region.

Power amplifier's AM-AM and AM-PM diagrams that are simulated in wide range of $V_{\text {in }}$ to shows nonlinear region are indicated in Figure 1. Also, the figure shows fitting diagram with different color that is used to calculate power amplifier's transfer function complex coefficients.

To obtain frequency function coefficients, 10 tests in ADS with previous condition are performed with different input frequency in each test and complex coefficients for each test are obtained with the method that is described. Input frequency varies between $(850+\mathrm{B}) \mathrm{MHz}$ and $(850-\mathrm{B}) \mathrm{MHz}$ to fill the range $(\mathrm{B}=0.62 \mathrm{MHz})$. After these tests, 10 complex values for each coefficient are acquired. Hence, each coefficient with the mean of these 10 values has the equation which frequency is itsvariable.

To validate our analysis, with the mean of our equations and coefficients we can draw $\mathrm{IM}_{1,3,5}$ diagrams (amplitude and phase) as a function of input amplitude of two-tone test " $s$ ". We compare these diagrams with those we get from two-tone test of our power amplifier that simulated in ADS. For considering frequency effect in our analysis, we must posit our coefficients $a_{2 n+1}$ as a function of frequency. 


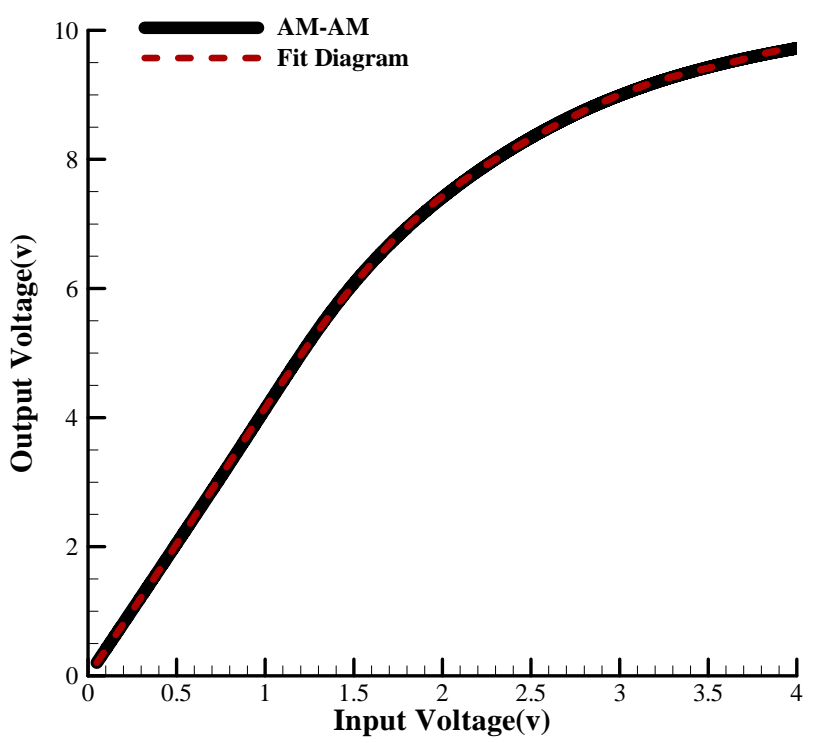

(a)

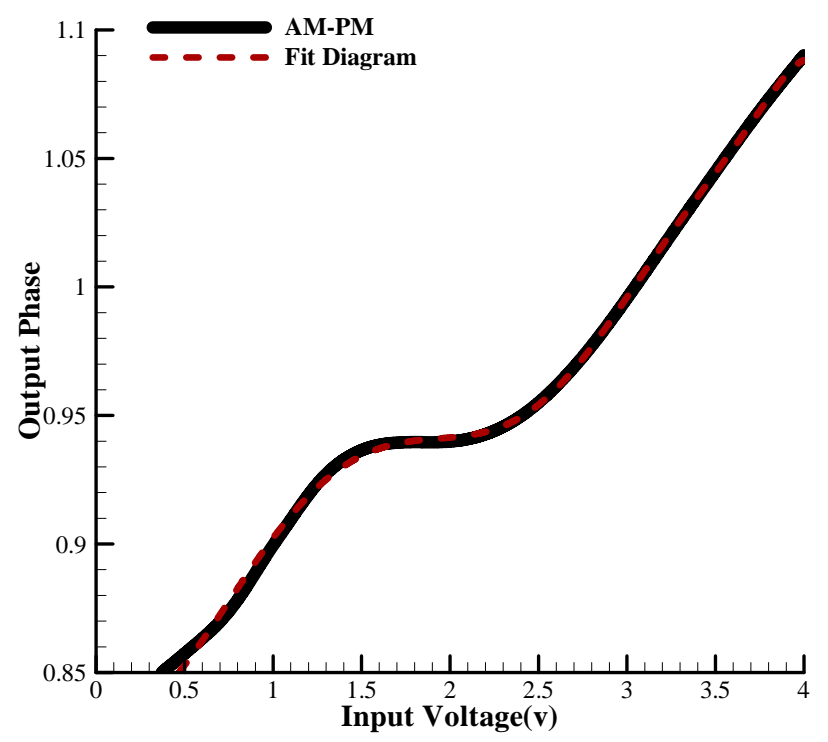

(b)

Figure 1. (a) Amplitude to amplitude (AM-AM) and (b) Magnitude to phase of output in compare with diagram that fitted in MATLAB.

Result of our simulation in compare with analytical expression (with or without considering frequency effect) for $\mathrm{IM}_{1,3,5}$ (phase and amplitude) are plotted in Figures 2-4. In all figures, diagrams that frequency effect isconsidered in their $a_{2 n+1}$ coefficients (blue), show better fit to simulated results (red) than the other one (green). In Equations (8) and (11), it is obvious that every coefficient related to one type of nonlinearity or are the dominate factor in them. For example $\mathrm{a}_{1}$ is the dominate factor in $\mathrm{IM}_{1}$. It shows that little variation in its value makes huge change in predicting $\mathrm{IM}_{1}$. Without any doubt, errors that occurred in fitting make some errors in calculating

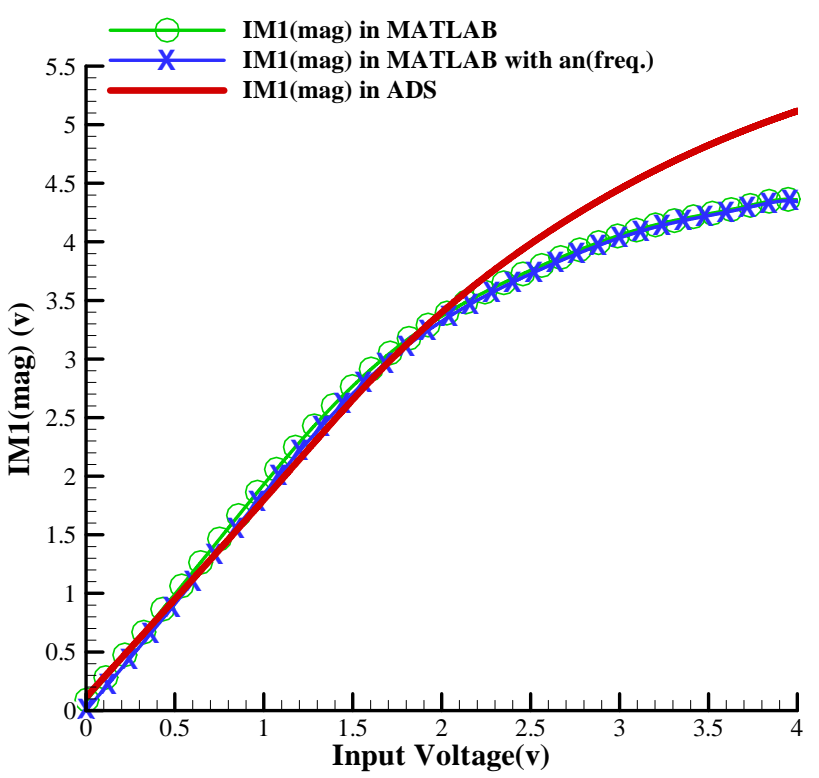

(a)

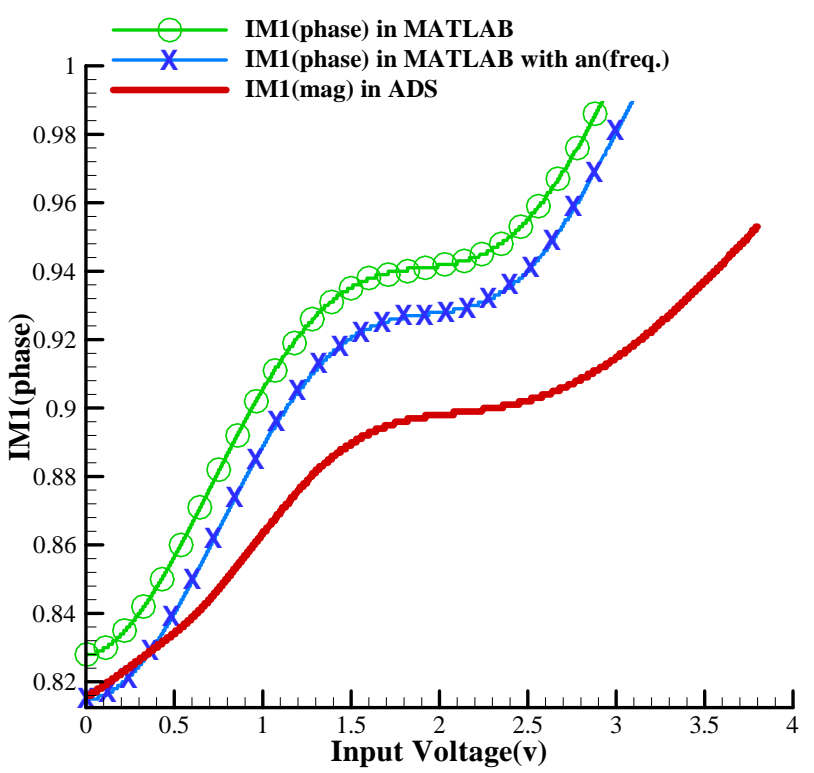

(b)

Figure 2. (a) Magnitude and (b) Phase of $I M_{1}$ in compare with analytical expression that plotted in MATLAB.

coefficients $a_{2 n+1}$.

As we can see in Figure 2, the difference between predicted and simulated results is due to the $a_{2 n+1}$ but difference in $\mathrm{IM}_{1}$ (magnitude or phase) is higher than $\mathrm{IM}_{3}$ or $\mathrm{IM}_{5}$. The cause of this error is due to the higher value of $a_{1}$, that is the dominate factor in calculating $\mathrm{IM}_{1}$, than the other factors. The coefficient $a_{1}$ is related to the linear gain $\mathrm{G}$ of the amplifier, and the coefficients $a_{3}$ and $a_{5}$, that are the dominate factors in calculating $\mathrm{IM}_{3}$ and $\mathrm{IM}_{5}$, respectively, are directly related to $\mathrm{IP}_{3}$ and $\mathrm{IP}_{5}$. Because of distortion nature of $a_{3}$ and $a_{5}$ it is obvious that 


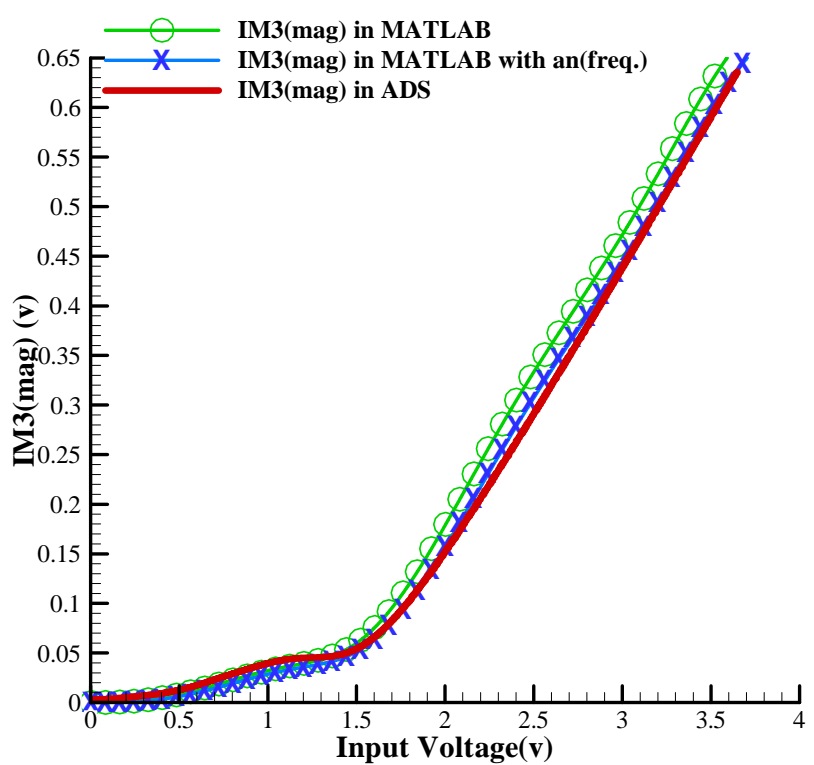

(a)

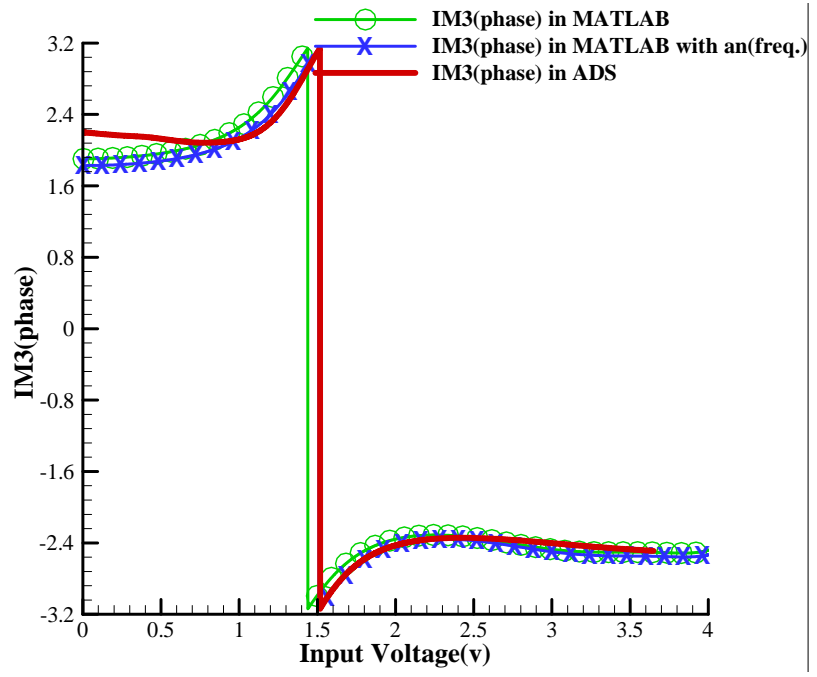

(b)

Figure 3. (a) Magnitude and (b) Phase of $\mathrm{IM}_{3}$ in compare with analytical expression that plotted in MATLAB.

they have smaller values than $a_{1}$ and, so, error in $a_{1}$ shows higher difference than errors in $a_{3}$ and $a_{5}$, as we can see in Figures 3 and 4.

Totally, results show that both magnitude and phase of each frequency (each IM) are predicted well by Equation (11), and have better match if we consider frequency effect. Though we did not compare it with real measurement results, HB (Harmonic Balance) simulation in ADS is known as the most accurate simulation method for a real system.

\section{Conclusion}

In this paper, we proposed a method to predict inter mo-

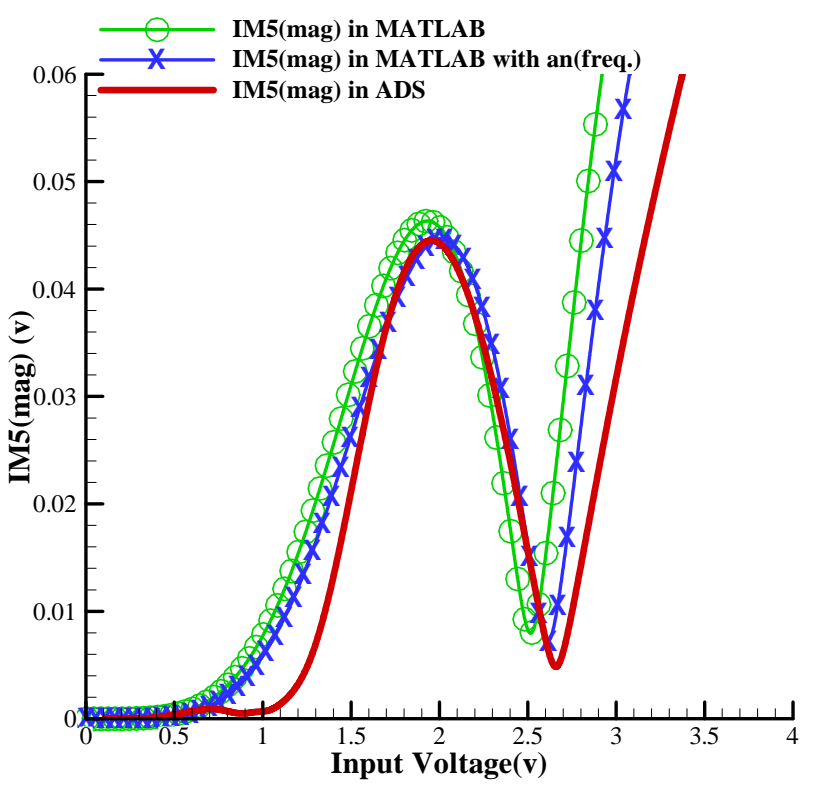

(a)

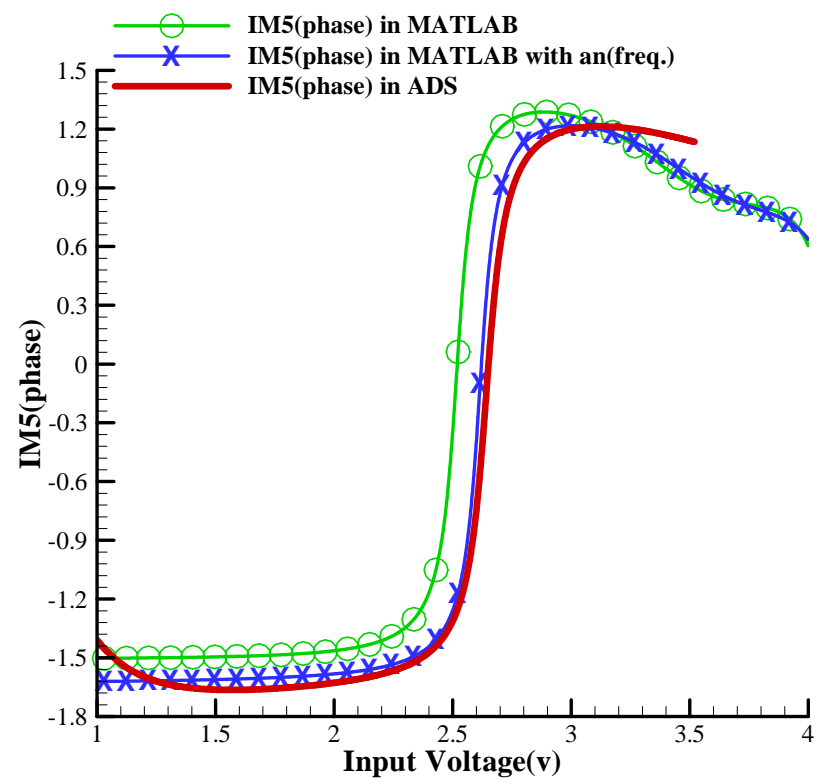

(b)

Figure 4. (a) Magnitude and (b) Phase of $\mathrm{IM}_{5}$ in compare with analytical expression that plotted in MATLAB.

dulation (IM) products based on AM-AM and AM-PM diagrams of the power amplifier. Our method shows not only input magnitude but also input frequency is affected output IM products. With the mean of this method and considering frequency effect in it, it is possible to predict IM products for wide band frequency range input, and input with complexity in their phase or frequency. In addition there is no need for circuit details of our power amplifier in our analyzing. Simulated results of power amplifier in compare with results that are gained from our method show good fitting with each other that shows 
our method is accurate.

\section{REFERENCES}

[1] R. Raich, H. Qian and G. T. Zhou, “Orthogonal Polynomials for Power Amplifier Modeling and Predistorter Design," IEEE Transactions on Vehicular Technology, Vol. 53, No. 5, 2004, pp. 1468-1479. doi:10.1109/TVT.2004.832415

[2] B. Larkin, "Multiple-Signal Intermodulation and Stability Consideration in the Use of Linear Repeaters," Proceeding of 41st IEEE Vehicular Technology, St. Louis, 19-22 May 1991, pp. 747-752.

[3] Q. Wu, M. Testa and R. Larkin, "Linear RF Power Amplifier Design for CDMA Signal,” IEEE MIT-S Digest, Corvallis, June 1996, pp. 851-854.

[4] G. T. Zhou and J. S. Kenney, "Predicting Spectral Regrowth of Nonlinear Power Amplifiers,” IEEE Transactions on Communications, Vol. 50, No. 5, 2002, pp. 718722. doi:10.1109/TCOMM.2002.1006553

[5] C. H. Tseng, "Estimation of Cubic Nonlinear Bandpass Channels in Orthogonal Frequency-Division Multiplexing Systems,” IEEE Transaction on Communications, Vol. 58, No. 5, 2010, pp. 1415-1425. doi:10.1109/TCOMM.2010.05.080573

[6] M. E. Gadringer, C. Schuberth and G. Magerl "Behavioral Modeling of the Frequency Translation Process in Direct Conversion Transmitters," International Journal of $R F$ and Microwave Computer-Aided Engineering, Vol. 20, No. 3, 2010, pp. 347-359. doi:10.1002/mmce.20439

[7] S. J. Yi, S. Nam, S. H. Oh and J. H. Han, "Prediction of a CDMA Output Spectrum Based on Intermodulation Products of Two-Tone Test," IEEE Transaction on Microwave Theory and Techniques, Vol. 49, No. 5, 2001, pp. 767946. doi:10.1109/22.920152

[8] A. H. Coskun and S. Demir, "A Mathematical Characterization and Analysis of a Feed forward Circuit for CDMA Applications," IEEE Transaction on Microwave Theory and Techniques, Vol. 51, No. 3, 2003, pp. 767777. doi:10.1109/TMTT.2003.808582

[9] C. Liu, H. Xiao, Q. Wu and F. Lit, "Linear RF Power Amplifier Design for Wireless Signals: A Spectrum Analysis Approach,” International Conference on Acoustics, Speech, and Signal Processing, Vol. 4, 2003, pp. 568571.

[10] A. M. A. Hemmatyar and F. Farzaneh, "Predicting of InterModulation Rejection Values for the First and Second Adjacent Channels in Feed-Forward Linearised Microwave Amplifiers Using Closed-Form Expressions," IET Microwaves Antennas \& Propagation, Vol. 1, No. 3, 2007, pp. 782-789. doi:10.1049/iet-map:20060342

[11] M. L. Ku, S. H. Lu, L. C. Wang and S. H. Yan, "Nonlinear Effect of Receiver Amplifier for $60 \mathrm{GHz}$ Radio Communication," IEEE International Conference on Vehicular Technology, 5-8 September 2011, pp. 1-5.
doi:10.1109/VETECF.2011.6093112

[12] K. G. Gard, H. M. Gutierrez and M. B. Steer, "Characterization of Spectral Regrowth in Microwave Amplifiers Based on the Nonlinear Transformation of a Complex Gaussian Process," IEEE Transaction on Microwave Theory and Techniques, Vol. 47, No. 7, 1999, pp. 1059-1069. doi:10.1109/22.775437

[13] G. T. Zhou, "Analysis of Spectral Regrowth of Weakly Nonlinear Power Amplifiers," IEEE Communication Letters, Vol. 4, No. 11, 2000, pp. 357-359.

[14] C. Liu, H. Xiao, Q. Wu and F. Li, "Linear RF Power Amplifier Design for TDMA Signals: A Spectrum Analysis Approach,” Proceedings of International Conference on Acoustics, Speech, and Signal Processing, Salt Lake City, Vol. 4, May 2001, pp. 2665-2668. doi:10.1109/ICASSP.2001.940550

[15] M. M. Rahmati, A. Abdipour, A. Mohammadi and G. Moradi, "An Analytic Approach for CDMA Output of Feed forward Power Amplifier," Analog Integrated Circuit and Signal Processing, Vol. 66, No. 3, 2011, pp. 349361.

[16] E. Cottais, Y. Wang and S. Toutain, "Spectral Regrowth at the Output of a Memoryless Power Amplifier with Multicarrier Signals," IEEE Transactions on Communications, Vol. 56, No. 7, 2008, pp. 1111-1118. doi:10.1109/TCOMM.2008.050149

[17] J. P. Aikio and T. Rahkonen, “A Comprehensive Analysis of AM-AM and AM-PM Conversion in an LDMOS RF Power Amplifier," IEEE Transactions on Microwave Theory and Techniques, Vol. 57, No. 2, 2009, pp. 262-270. doi:10.1109/TMTT.2008.2011161

[18] S. Meza, M. O’Droma, Y. Lei and A. Goacher, “Some New Memory Less Behavioral Models of Wireless Transmitter Solid State Power Amplifiers,” IEEE Automation, Quality and Testing, Robotics, Vol. 1, 2008, pp. 96-98. doi:10.1109/AQTR.2008.4588714

[19] M. Masood, J. Staudinger, J. Wood, M. Bokatius and J. S. Kenney, "Linearity Considerations for a High Power Doherty Amplifier," IEEE International Conference on Power Amplifier for Wireless and Radio Applications, Atlanta, 15-18 January 2012, pp. 77-80. doi:10.1109/PAWR.2012.6174912

[20] L. W. Couch, "Digital and Analog Communication Systems,” Prentice-Hall Inc., Upper Saddle River, 1996.

[21] T. S. Rappaport, "Wireless Communication Principle and Practice,” Prentice-Hall Inc., Upper Saddle River, 1996.

[22] M. C. Jeruchim, P. Balabon and K. S. Shanmugan, "Simulation of Communication Systems,” Kluwer Academic Publishers, Springer, New York, 2002.

[23] A. Chatterjee, S. Devarakond and S. Sen, "Phase Distortion to Amplitude Conversion-Based Low-Cost Measurement of AM-AM and AM-PM Effects in RF Power Amplifiers," IEEE Transaction on Very Large Scale Integration (VLSI) Systems, Vol. PP, No. 99, 2011, pp. 1-13. doi:10.1109/TVLSI.2011.2160376 